\title{
Critical role of the circadian clock in memory formation: lessons from Aplysia
}

\section{Lisa C. Lyons*}

Department of Biological Science, Program in Neuroscience, Florida State University, Tallahassee, FL, USA

\section{Edited by:}

Oliver Rawashdeh, Goethe University

Frankfurt, Germany

\section{Reviewed by:}

Oliver Rawashdeh, Goethe University

Frankfurt, Germany

Avraham Susswein, Bar-llan

University, Israel

*Correspondence:

Lisa C. Lyons, Department of Biological Science, Florida State

University, 319 Stadium Drive,

Tallahassee, FL 32306-4295, USA.

e-mail:lyons@bio.fsu.edu
Unraveling the complexities of learning and the formation of memory requires identification of the cellular and molecular processes through which neural plasticity arises as well as recognition of the conditions or factors through which those processes are modulated. With its relatively simple nervous system, the marine mollusk Aplysia californica has proven an outstanding model system for studies of memory formation and identification of the molecular mechanisms underlying learned behaviors, including classical and operant associative learning paradigms and non-associative behaviors. In vivo behavioral studies in Aplysia have significantly furthered our understanding of how the endogenous circadian clock modulates memory formation. Sensitization of the tail-siphon withdrawal reflex represents a defensive non-associative learned behavior for which the circadian clock strongly modulates intermediate and long-term memory formation. Likewise, Aplysia exhibit circadian rhythms in long-term memory, but not short-term memory, for an operant associative learning paradigm. This review focuses on circadian modulation of intermediate and long-term memory and the putative mechanisms through which this modulation occurs. Additionally, potential functions and the adaptive advantages of time of day pressure on memory formation are considered. The influence of the circadian clock on learning and memory crosses distant phylogeny highlighting the evolutionary importance of the circadian clock on metabolic, physiological, and behavioral processes. Thus, studies in a simple invertebrate model system have and will continue to provide critical mechanistic insights to complementary processes in higher organisms.

Keywords: learning and memory, circadian rhythms, Aplysia, sensitization, operant learning

\section{APLYSIA AS A MODEL FOR CIRCADIAN RESEARCH}

Although less familiar to current students of circadian biology, Aplysia californica represents one of the earliest models used to systematically study the circadian clock. Members of the genus Aplysia, frequently referred to as sea hares due to the appearance of the upward projecting rhinophores, are marine mollusks from the family Aplysiidae (class: Gastropoda, order: Opisthobranchia). While the fossil record for Aplysia species is limited, the genus Akera, a sister taxon to Aplysiidae representing the primitive sea hare, appears in the fossil record as early as 165 mya (reviewed in Medina and Walsh, 2000; Medina et al., 2001). Aplysia species likely diverged considerably later, perhaps as recently as 25 mya during the Miocene period (reviewed in Medina and Walsh, 2000; Medina et al., 2001). Aplysia are found throughout the world generally in warm waters with the diurnal $A$. californica (northeast Pacific), nocturnal A. fasciata (Mediterranean) and A. kurodai (northwestern Pacific), commonly used in neuroscience. Aplysia are hermaphroditic animals, although not self-fertilizing, that budget their time for reproductive, feeding, and exploratory activities (Susswein et al., 1983; Carefoot, 1989; Ziv et al., 1991a,b). Feeding on algae, Aplysia inhabit the photic zone, primarily the intertidal and sub-littoral zones commonly at depths less than $20 \mathrm{~m}$ (Kandel, 1979), and are influenced by daily light-dark cycles. Aplysia exhibit robust circadian rhythms in locomotor activity
(Strumwasser, 1973) and feeding behavior (Kupfermann, 1974; Levenson et al., 1999).

Groundbreaking research determined that the isolated Aplysia eye ( $<1 \mathrm{~mm}$ in size) contained all necessary components of a circadian system: entrainment, oscillator, and outputs (Jacklet, 1969; Eskin, 1971). Isolated eyes demonstrate free-running circadian rhythms in optic nerve impulses that can be entrained and phaseshifted (Jacklet, 1969; Eskin, 1971). Ocular circadian rhythms can also be recorded in vivo (Block, 1981). Numerous studies in Aplysia outlined the necessity of transcription (Raju et al., 1991; Koumenis et al., 1996) and translation (Rothman and Strumwasser, 1976, 1977; Jacklet, 1977; Lotshaw and Jacklet, 1986; Yeung and Eskin, 1987) for circadian oscillator function. Early studies using the isolated eye system identified second messenger signaling and the effectiveness of neurotransmitters in phase-shifting the oscillator (Corrent and Eskin, 1982; Corrent et al., 1982; Eskin et al., 1982; Eskin and Takahashi, 1983; Colwell et al., 1992) as well as the necessity of protein synthesis for phase-shifts (Eskin et al., 1984; Raju et al., 1990).

Despite a half-century of circadian research in Aplysia, the molecular circuitry of the Aplysia circadian system has not been fully elucidated. The Aplysia central nervous system consists of about 20,000 neurons organized into discrete ganglia. Circadian oscillatory neurons localizing to the base of the eye likely serve as central pacemakers with the eyes also serving in primary 
photoentrainment. Ocular oscillators send afferent fibers via the optic nerve to central nervous system ganglia excluding the buccal ganglia (Herman and Strumwasser, 1984; Olson and Jacklet, 1985). Extraocular photoreceptors present in rhinophores and anterior tentacles can affect circadian locomotor activity (Block and Lickey, 1973; Roberts and Block, 1982). The cerebral ganglion also contains photoreceptors and may serve as a point of convergence for photic information (Eskin, 1971; Block and Smith, 1973; Roberts and Block, 1982). As the molecular components of the oscillator remain unknown, potentially peripheral circadian oscillators contain light sensitive molecules that function in entrainment as observed in Drosophila and zebrafish (Plautz et al., 1997; Whitmore et al., 2000).

In the intact animal, ocular circadian oscillatory neurons strongly influence circadian locomotor activity with removal of the eyes resulting in arrhythmicity in most animals (Strumwasser, 1973; Lickey etal., 1976, 1977, 1983). However, the eyes are not strictly necessary for circadian activity as some eyeless animals continue to demonstrate rhythmic locomotor activity (Block and Lickey, 1973; Strumwasser, 1973; Lickey et al., 1977) suggesting the existence of extraocular pacemakers in addition to extraocular photoreceptors. Little progress has been made in identifying pacemakers in central nervous system ganglia. Although the abdominal ganglion is unnecessary for circadian activity rhythms (Strumwasser etal., 1972), early research described abdominal neuron R15 as a candidate for a circadian pacemaker (Strumwasser, 1965) entrained by light-dark cycles (Lickey, 1969) with diurnal and circadian rhythms in spiking activity (Audesirk and Strumwasser, 1975; Woodson and Schlapfer, 1979). However, sustained firing rhythms appear absent under prolonged constant conditions (Lickey et al., 1976). The cerebral ganglion appears a likely location for central nervous system circadian pacemaker neurons with its integration of multiple photic inputs and numerous connections to other ganglia (Weiss et al., 1978; Rosen et al., 1991; Wright et al., 1995). Moreover, cerebral ganglion neurons send efferent connections to ocular pacemaker neurons (Olson and Jacklet, 1985; Takahashi et al., 1989) potentially providing a mechanism for central nervous system feedback.

Although the components of the core circadian oscillator in Aplysia remain unknown, the circadian gene period has been cloned in the closely related mollusk Bulla (Constance et al., 2002). Unusually bPer mRNA and protein levels were shown to cycle only in light-dark cycles in retinal pacemakers with no oscillations observed in constant conditions (Constance et al., 2002). The only identified Aplysia gene for which rhythmic expression has been shown is the immediate early gene $A p C / E B P$, a leucine $\beta$ Zip transcription factor, which shows significantly higher mRNA and protein levels during the night (Hattar et al., 2002; Lyons et al., 2006b). Whilst ApC/EBP appears unlikely to function in the core oscillator, circadian rhythms in protein abundance in cerebral and pleural ganglia indicate that the circadian clock impacts the central nervous system.

\section{CIRCADIAN MODULATION OF LEARNING AND MEMORY}

The circadian clock imparts a powerful adaptive advantage to organisms through the coordination of metabolic and physiological events in anticipation of regular environmental occurrences.
Aplysia represents an excellent system for investigating circadian modulation of memory given the simplicity of its neuronal organization combined with numerous in vivo and in vitro learning paradigms that have made Aplysia a favorite model organism for neuroscientists. The most well-studied learning paradigms concentrate on defensive withdrawal reflexes using non-associative sensitization and habituation, and classical conditioning of withdrawal reflexes (reviewed in Bailey et al., 2008). In addition to defensive reflexes, the neural circuitry of the feeding system exhibits a high degree of plasticity and is frequently studied using operant and classical conditioning paradigms (reviewed in Elliott and Susswein, 2002; Baxter and Byrne, 2006).

Initial research in Aplysia examining circadian modulation of memory investigated sensitization of the siphon-withdrawal reflex, non-associative learning in which application of a noxious stimulus enhances subsequent responses to a mild stimulus. In light-dark cycles and constant conditions, animals exhibited robust long-term memory when training was administered during the (subjective) day (Fernandez et al., 2003; Lyons et al., 2006b). However, when training was performed at night, little longterm sensitization was observed. Potentially, circadian regulation of memory formation occurs through gating sensory perception during initial reception or transmission of sensory stimuli. Pretraining baseline responses in the threshold stimulus necessary to elicit siphon withdrawal and withdrawal duration were not circadianly regulated suggesting that the circadian clock modulated memory rather than sensory perception. In later studies, diurnal and circadian rhythms also were observed for intermediate-term sensitization (Lyons et al., 2008), a form of memory dependent upon protein synthesis but not transcription (Sutton et al., 2001; Sutton and Carew, 2002).

Circadian modulation of memory has also been investigated using a more complex operant learning paradigm, learning that food is inedible (LFI). In this paradigm, the animal is presented with netted seaweed that cannot be swallowed to which the animal responds with repeated cycles of biting, swallowing attempts, and rejection of the netted food (Susswein and Schwarz, 1983; Schwarz and Susswein, 1986). An association is formed between the failure of swallowing attempts for feeding and the specific seaweed (Susswein et al., 1986; Michel et al., 2011a). As with sensitization, long-term LFI memory appears strongly regulated by the circadian clock. Animals that are trained during the (subjective) day demonstrate robust memory $24 \mathrm{~h}$ later, whereas animals trained at night display virtually no long-term memory with responses similar to naïve animals (Lyons et al., 2005, 2006a). Thus, the circadian clock strongly modulates non-associative and associative long-term memory in Aplysia.

For long-term sensitization and LFI memory, circadian modulation targets memory formation rather than recall. Animals trained during the day demonstrated robust memory even when tested 30-36 h later during the following night (Fernandez et al., 2003; Lyons et al., 2005). However, animals trained during the night showed little long-term memory at any time point. These results stand in contrast to hippocampal dependent contextual fear conditioning in which the strength of memory recall appears to be regulated by the circadian clock (Chaudhury and Colwell, 2002). 
Where are the oscillators responsible for modulation of memory? As with locomotor activity, pacemaker neurons located in the eyes do not appear necessary for entrainment or maintenance of circadian modulation of memory in Aplysia. Eyeless animals exhibit circadian rhythms in long-term memory for sensitization and LFI (Lyons et al., 2006a). Furthermore, animals re-entrained after eye removal to a new light-dark cycle exhibited significant circadian rhythms in long-term memory in phase with the re-entrained cycle (Lyons et al., 2006a). These experiments support the hypothesis that central nervous system ganglia contain independent circadian oscillators that can be entrained through extraocular photoreceptors. While the neurons responsible for memory may not be oscillators themselves, mechanisms exist through which hypothetical pacemaker cells located in the cerebral ganglion could modulate the activity of neurons involved in sensitization and LFI memory circuits.

\section{POSSIBLE MECHANISMS OF MODULATION}

Learning and memory can be diagrammed linearly with sensory perception, learning, memory formation, and recall occurring sequentially. Memory formation encompasses all molecular and cellular changes necessary to maintain learned behavior (Sweatt, 2010) and can be subdivided into induction, molecular consolidation involving transcription and translation, and maintenance. For Aplysia, it appears that memory formation rather than sensory perception or recall is the primary process modulated by the circadian clock as time of training appears to be the determinant factor. However, short-term memory for sensitization and LFI is independent of the circadian cycle (Fernandez et al., 2003; Lyons et al., 2005) suggesting that early steps common to short, intermediate, and long-term memory may not be primary targets for circadian regulation.

What are potential mechanisms through which the circadian clock modulates memory formation? Sensitization and LFI memory involve distinct neural and molecular circuits (Cleary et al., 1998; Elliott and Susswein, 2002; Bristol et al., 2004; Cropper et al., 2004; Bailey and Kandel, 2008). During sensitization, serotonin release by cerebral ganglion facilitatory neurons initiates a sequence of events resulting in pre-synaptic facilitation of pleural sensory neurons and post-synaptic changes in pedal motor neurons (Villareal et al., 2007; Bailey et al., 2008; Cai et al., 2008). Early induction steps include amplification of adenylyl cyclase activity, increased cAMP levels and PKA activation (Kandel, 2001; Reissner et al., 2006). Sensitization training induces serotonin release into the hemolymph with increased training resulting in greater levels of serotonin (Marinesco and Carew, 2002; Marinesco et al., 2004). Serotonin release following sensitization training is circadianly regulated (Lyons et al., 2006b) providing one mechanism through which the circadian clock modulates intermediate and long-term sensitization. Although serotonin induced activation of cAMP-PKA signaling is common to short and long-term memory, potentially circadian regulation of persistent PKA activity and subsequent CREB-dependent transcription occurs for long-term memory. During long-term facilitation, an in vitro correlate for sensitization, persistent PKA activity and CREB activation induce necessary expression of ApC/EBP (Alberini et al., 1994; Muller and Carew, 1998). The circadian clock regulates training-induced
$A p C / E B P$ expression with sensitization training during the day, but not the night, resulting in multi-fold increases in protein abundance (Lyons et al., 2006b). Persistent PKA activation also is necessary for long-term LFI memory and LFI training results in increased $A p C / E B P$ mRNA and protein abundance in the buccal ganglia (Levitan et al., 2008; Michel et al., 2011a). Potentially, circadian modulation of LFI memory also occurs through regulation of the CAMP-PKA-CREB pathway.

There appear to be distinct processes in separate neuronal clusters modulated by the circadian clock during sensitization. Circadian modulation of sensitization also occurs downstream of serotonin release from facilitatory neurons. In experiments in which in vivo sensitization was induced using serotonin, animals exhibited long-term sensitization with a robust circadian rhythm similar in phase and amplitude to previous studies (Lyons et al., 2006b). This suggests that circadian modulation occurs either pre-synaptically or post-synaptically through a mechanism independent of rhythmic serotonin release.

The MAPK signaling cascade appears a likely target for circadian modulation in sensitization and potentially for LFI memory as MAPK also acts as an integrator of neuronal inputs. Intermediate and long-term sensitization, but not short-term memory, are dependent upon training-induced MAPK activation and prolonged MAPK signaling (Martin et al., 1997; Michael et al., 1998; Sharma etal., 2003; Sharma and Carew, 2004). Overall basal MAPK activity in pleural and cerebral ganglia does not appear to be rhythmically regulated. Following sensitization training during the (subjective) day, but not the night, levels of phosphoMAPK are greatly increased in pleural ganglia (Lyons et al., 2006b). While the circadian induction of MAPK activation has not been examined for LFI memory, a prolonged MAPK signaling phase in the buccal ganglia appears necessary for long-term, but not short-term, LFI memory (Michel et al., 2011b). The dual circadian modulation of learning-induced activation of MAPK and $A p C / E B P$ expression may be sufficient to explain circadian modulation of long-term sensitization and LFI memory.

Just as the mechanisms involved in memory formation occur through a network of interconnected signaling pathways, it seems unlikely that circadian modulation of memory occurs through simple linear connections. More realistically, circadian modulation affects a network of signaling pathways resulting in dynamic regulation of behavior. Although the extent of circadian regulation of gene expression or signaling has not been investigated in Aplysia, genome wide expression studies in other organisms suggests that a considerable portion of the genome is under circadian regulation (reviewed in Doherty and Kay, 2010). Given the disparate anatomical and molecular circuitry for sensitization and LFI memory, humoral factors may also serve as a means for circadian regulation of multiple neural circuits.

\section{FUNCTION OF CIRCADIAN MODULATION OF MEMORY}

From an evolutionary perspective, if no function or selective advantage to circadian modulation of memory existed then one would predict considerable laxity in regulation. The observance of circadian influences on memory across phylogeny and apparent multiple levels of regulation as observed in Aplysia suggest specific functions for circadian modulation. Alternatively, 
the synchronized phase regulation of memory could occur as a byproduct of broader circadian regulation of cellular and metabolic processes.

Given the relatively small number of neurons in the central nervous system in Aplysia, wide-ranging circadian regulation of transcription, translation, or kinase signaling pathways seems feasible. Potentially, regulation of memory occurs alongside functional regulation of other processes and behaviors. If cellular processes for feeding or locomotion are circadianly regulated and these same signaling pathways or neurons are involved in learning, memory formation may be coincidentally modulated. Several lines of evidence suggest that circadian modulation of memory is not a consequence of general circadian regulation, although this does not preclude functional coordination of behavior and memory through cooperative regulatory mechanisms. (1) Signaling pathways involved in memory do not appear to exhibit widespread basal circadian activity, at least for MAPK activity in whole ganglia. If circadian regulation of memory was due to coincident regulation of MAPK signaling, one would expect basal MAPK activity levels to be circadianly regulated. (2) Multiple neuron types and distinct pathways appear targeted by circadian regulation during sensitization. (3) Circadian dysfunction induces decrements in memory formation in animal models and humans (Cho et al., 2000; Cho, 2001; Ruby et al., 2008; Loh et al., 2010). Thus, circadian modulation of memory appears to represent purposeful regulation that may be phase coordinated with behavioral activities as for A. californica. One would expect a nocturnal Aplysia species to demonstrate greater memory during the night. This is indeed the case as observed for long-term LFI memory in the nocturnal $A$. fasciata (Lyons et al., 2005).

Intermediate and long-term memory exact a metabolic energy cost through requirements for persistent kinase activity, protein synthesis, and gene expression. Coordination of memory formation with an animal's activity allows for the orchestrated circadian downregulation of neuronal activity during periods of rest. Sleep has been postulated as functioning in synaptic homeostasis by reducing synaptic strength to baseline levels enabling energy savings (Tononi and Cirelli, 2006; Hanlon et al., 2011).

\section{REFERENCES}

Alberini, C. M., Ghirardi, M., Metz, R., and Kandel, E. R. (1994). C/EBP is an immediate-early gene required for the consolidation of long-term facilitation in Aplysia. Cell 76, 1099-1114.

Audesirk, G., and Strumwasser, F. (1975). Circadian rhythm of neuron R15 of Aplysia californica: in vivo photoentrainment. Proc. Natl. Acad. Sci. U.S.A. 72, 2408-2412.

Bailey, C. H., Barco, A., Hawkins, R. D., Kandel, E. R., and Byrne, J. H. (2008). "Molecular studies of learning and memory in Aplysia and the hippocampus: a comparative analysis of implicit and explicit memory storage," in Learning and Memory: A Comprehensive Reference, ed. J. H. Byrne (Oxford: Academic), 11-29.
Bailey, C. H., and Kandel, E. R. (2008). Synaptic remodeling, synaptic growth and the storage of long-term memory in Aplysia. Prog. Brain Res. 169, 179-198.

Baxter, D. A., and Byrne, J. H. (2006). system for comparing cellular mechanisms of classical and operant conditioning. Learn. Mem. 13, 669-680.

Block, G. D. (1981). In vivo recording of the ocular circadian rhythm in Aplysia. Brain Res. 222, 138-143.

Block, G. D., and Lickey, M. E. (1973). Extraocular photoreceptors and oscillators can control the circadian rhythm of behavioral activity in Aplysia. J. Comp. Physiol. 84, 367-374.

Block, G. D., and Smith, J. T. (1973). Cerebral photoreceptors in Aplysia. Feeding behavior of Aplysia: a model

Sleep dependent reductions in synaptic markers, synaptic strength, synaptic branching, and gene expression were recently shown in Drosophila (Cirelli et al., 2005; Gilestro et al., 2009; Bushey et al., 2011). While A. californica sleep has not been examined, circadian interactions between regulation and the homeostatic need for rest are likely. Intermediate and long-term memory formation at night with associated energy demands would be contraindicated with the synaptic homeostasis hypothesis. However, the coordination of intermediate and long-term memory formation with behavioral activities does not preclude the animal's response to immediate crises or dire situations. Short-term memory in Aplysia is not circadianly regulated allowing for increased defenses in response to predators or differentiation between edible and inedible foods to meet an immediate feeding need.

\section{FUTURE DIRECTIONS}

Across phylogeny most studies of circadian modulation of memory employ negatively reinforced learning paradigms such as fear conditioning (Valentinuzzi et al., 2001; Chaudhury and Colwell, 2002), conditioned taste aversion (Manrique et al., 2004; GomezSerrano et al., 2009), conditioned place avoidance (Rawashdeh et al., 2007), and olfactory conditioning (Lyons and Roman, 2009). Negatively reinforced paradigms produce robust learning with a greater dynamic range in memory strength possible compared to positively reinforced paradigms allowing for increased sensitivity in quantifying modulation. Understanding the function(s) of circadian modulation of memory will require a broader perspective with additional research investigating positively reinforced learning paradigms. The detailed neurocircuitry and reductionist approaches available in Aplysia suggest future research will provide continuing mechanistic insight into circadian modulation of memory validating the usefulness of simple invertebrate models for understanding evolutionarily conserved system interactions.

\section{ACKNOWLEDGMENTS}

The research was supported by National Institute of Mental Health grant R01MH81012. I would like to thank Dr. Maximilian Michel for thoughtful discussions and critical reading of the manuscript.

Comp. Biochem. Physiol. A 46, 115-121.

Bristol, A. S., Sutton, M. A., and Carew, T. J. (2004). Neural circuit of tail-elicited siphon withdrawal in Aplysia. I. Differential lateralization of sensitization and dishabituation. J. Neurophysiol. 91, 666-677.

Bushey, D., Tononi, G., and Cirelli, C. (2011). Sleep and synaptic homeostasis: structural evidence in Drosophila. Science 332, 1576-1581.

Cai, D., Chen, S., and Glanzman, D. L. (2008). Postsynaptic regulation of long-term facilitation in Aplysia. Curr. Biol. 18, 920-925.

Carefoot, T. H. (1989). A comparison of time/energy budgeting in two species of tropical sea hares Aplysia. J. Exp. Mar. Biol. Ecol. 131, 267-282.
Chaudhury, D., and Colwell, C. S. (2002). Circadian modulation of learning and memory in fearconditioned mice. Behav. Brain Res. 133, 95-108.

Cho, K. (2001). Chronic 'jet lag' produces temporal lobe atrophy and spatial cognitive deficits. Nat. Neurosci. 4, 567-568.

Cho, K., Ennaceur, A., Cole, J. C., and Suh, C. K. (2000). Chronic jet lag produces cognitive deficits. J. Neurosci. 20, RC66.

Cirelli, C., LaVaute, T. M., and Tononi, G. (2005). Sleep and wakefulness modulate gene expression in Drosophila. J. Neurochem. 94, 1411-1419.

Cleary, L. J., Lee, W. L., and Byrne, J. H. (1998). Cellular correlates of long-term sensitization in Aplysia. J. Neurosci. 18, 5988-5998. 
Colwell, C. S., Khalsa, S. B., and Block, G. D. (1992). FMRFamide modulates the action of phase shifting agents on the ocular circadian pacemakers of Aplysia and Bulla. J. Comp. Physiol. A 170, 211-215.

Constance, C. M., Green, C. B., Tei, H., and Block, G. D. (2002). Bulla gouldiana period exhibits unique regulation at the mRNA and protein levels. J. Biol. Rhythms 17, 413-427.

Corrent, G., and Eskin, A. (1982). Transmitterlike action of serotonin in phase shifting a rhythm from the Aplysia eye. Am. J. Physiol. 242, R333-R338.

Corrent, G., Eskin, A., and Kay, I. (1982). Entrainment of the circadian rhythm from the eye of Aplysia: role of serotonin. Am. J. Physiol. 242, R326-R332.

Cropper, E. C., Evans, C. G., Hurwitz, I., Jing, J., Proekt, A., Romero, A., and Rosen, S. C. (2004). Feeding neural networks in the mollusc Aplysia. Neurosignals 13, 70-86.

Doherty, C. J., and Kay, S. A. (2010). Circadian control of global gene expression patterns. Annu. Rev. Genet. 44, 419-444.

Elliott, C. J., and Susswein, A. J. (2002). Comparative neuroethology of feeding control in molluscs. J. Exp. Biol. 205, 877-896.

Eskin, A. (1971). Properties of the Aplysia visual system: in vitro entrainment of the circadian rhythm and centrifugal regulation of the eye. Z. Vergl. Physiol. 74, 353-371.

Eskin, A., Corrent, G., Lin, C. Y., and McAdoo, D. J. (1982). Mechanism for shifting the phase of a circadian rhythm by serotonin: involvement of cAMP. Proc. Natl. Acad. Sci. U.S.A. 79, 660-664.

Eskin, A., and Takahashi, J. S. (1983). Adenylate cyclase activation shifts the phase of a circadian pacemaker. Science 220, 82-84.

Eskin, A., Yeung, S. J., and Klass, M. R. (1984). Requirement for protein synthesis in the regulation of a circadian rhythm by serotonin. Proc. Natl. Acad. Sci. U.S.A. 81, 7637-7641.

Fernandez, R. I., Lyons, L. C., Levenson, J., Khabour, O., and Eskin, A. (2003). Circadian modulation of long-term sensitization in Aplysia. Proc. Natl. Acad. Sci. U.S.A. 100, 14415-14420.

Gilestro, G. F., Tononi, G., and Cirelli, C. (2009). Widespread changes in synaptic markers as a function of sleep and wakefulness in Drosophila. Science 324, 109-112.

Gomez-Serrano, M. A., Kearns, D. N., and Riley, A. L. (2009). The effects of light cycle phase on morphineinduced conditioned taste aversions in the Lewis, Fischer and SpragueDawley rat strains. Behav. Brain Res. 196, 116-122.

Hanlon, E. C., Vyazovskiy, V. V., Ugo, F., Tononi, G., and Cirelli, C. (2011). Synaptic potentiation and sleep need: clues from molecular and electrophysiological studies. Curr. Top. Med. Chem. 11, 2472-2482.

Hattar, S., Lyons, L. C., and Eskin, A. (2002). Circadian regulation of a transcription factor, ApC/EBP, in the eye of Aplysia californica. J. Neurochem. 83, 1401-1411.

Herman, K. G., and Strumwasser, F. (1984). Regional specializations in the eye of Aplysia, a neuronal circadian oscillator. J. Comp. Neurol. 230, 593-613.

Jacklet, J. W. (1969). A circadian rhythm of optic nerve impulses recorded in darkness from the isolated eye of Aplysia. Science 164, 563-564.

Jacklet, J. W. (1977). Neuronal circadian rhythm: phase shifting by a protein synthesis inhibitor. Science 198, 69-71.

Kandel, E. R. (1979). Behavioral Biology of Aplysia: A Contribution to the Comparative Study of Opistobranch Molluscs. San Francisco: W.H. Freeman and Company.

Kandel, E. R. (2001). The molecular biology of memory storage: a dialogue between genes and synapses. Science 294, 1030-1038.

Koumenis, C., Tran, Q., and Eskin, A. (1996). The use of a reversible transcription inhibitor, DRB, to investigate the involvement of specific proteins in the ocular circadian system of Aplysia. J. Biol. Rhythms 11, 45-56.

Kupfermann, I. (1974). Feeding behavior in Aplysia: a simple system for the study of motivation. Behav. Biol. 10, $1-26$.

Levenson, J., Byrne, J. H., and Eskin, A. (1999). Levels of serotonin in the hemolymph of Aplysia are modulated by light/dark cycles and sensitization training. J. Neurosci. 19, 8094-8103.

Levitan, D., Lyons, L. C., Perelman, A., Green, C. L., Motro, B., Eskin, A., and Susswein, A. J. (2008). Training with inedible food in Aplysia causes expression of C/EBP in the buccal but not cerebral ganglion. Learn. Mem. 15, 412-416.

Lickey, M. E. (1969). Seasonal modulation and non-24-hour entrainment of a circadian rhythm in a single neuron. J. Comp. Physiol. Psychol. 68, 9-17.

Lickey, M. E., Block, G. D., Hudson, D. J., and Smith, J. T. (1976). Circadian oscillators and photoreceptors in the gastropod, Aplysia. Photophysiology 23, 253-273.
Lickey, M. E., Hudson, D. J., and Hiaasen, S. O. (1983). Circadian organization in Aplysia: relations between locomotor rhythm and eye rhythms after cutting both, one or neither optic nerves. J. Comp. Physiol. A 153, 133-143.

Lickey, M. E., Wozniak, J. A., Block, G. D., Hudson, D. J., and Augter, G. K. (1977). The consequences of eye removal for the circadian rhythm of behavioral activity in Aplysia. J. Comp. Physiol. 118, 121-143.

Loh, D. H., Navarro, J., Hagopian, A., Wang, L. M., Deboer, T., and Colwell, C. S. (2010). Rapid changes in the light/dark cycle disrupt memory of conditioned fear in mice. PLoS ONE 5, e12546. doi: 10.1371/journal. pone. 0012546 .

Lotshaw, D. P., and Jacklet, J. W. (1986). Involvement of protein synthesis in circadian clock of Aplysia eye. Am. J. Physiol. 250, R5-R17.

Lyons, L. C., Rawashdeh, O., and Eskin, A. (2006a). Non-ocular circadian oscillators and photoreceptors modulate long term memory formation in Aplysia. J. Biol. Rhythms 21, 245-255.

Lyons, L. C., Collado, M. S., Khabour, O., Green, C. L., and Eskin, A. (2006b). The circadian clock modulates core steps in long-term memory formation in Aplysia. J. Neurosci. 26, 8662-8671.

Lyons, L. C., Green, C. L., and Eskin, A. (2008). Intermediate-term memory is modulated by the circadian clock. J. Biol. Rhythms 23, 538-542.

Lyons, L. C., Rawashdeh, O., Katzoff, A., Susswein, A. J., and Eskin, A. (2005). Circadian modulation of complex learning in diurnal and nocturnal Aplysia. Proc. Natl. Acad. Sci. U.S.A. 102, 12589-12594.

Lyons, L. C., and Roman, G. (2009). Circadian modulation of short-term memory in Drosophila. Learn. Mem. 16, 19-27.

Manrique, T., Molero, A., Ballesteros, M. A., Moron, I., Gallo, M., and Fenton, A. A. (2004). Time of day-dependent latent inhibition of conditioned taste aversions in rats. Neurobiol. Learn. Mem. 82, 77-80.

Marinesco, S., and Carew, T. J. (2002). Serotonin release evoked by tail nerve stimulation in the CNS of Aplysia: characterization and relationship to heterosynaptic plasticity. J. Neurosci. 22, 2299-2312.

Marinesco, S., Kolkman, K. E., and Carew, T. J. (2004). Serotonergic modulation in Aplysia. I. Distributed serotonergic network persistently activated by sensitizing stimuli. J. Neurophysiol. 92, 2468-2486.
Martin, K. C., Michael, D., Rose, J. C., Barad, M., Casadio, A., Zhu, H., and Kandel, E. R. (1997). MAP kinase translocates into the nucleus of the presynaptic cell and is required for long-term facilitation in Aplysia. Neuron 18, 899-912.

Medina, M., Collins, T. M., and Walsh, P. J. (2001). mtDNA ribosomal gene phylogeny of sea hares in the genus Aplysia (Gastropoda, Opisthobranchia, Anaspidea): implications for comparative neurobiology. Syst. Biol. 50, 676-688.

Medina, M., and Walsh, P. J. (2000). Molecular systematics of the order Anaspidea based on mitochondrial DNA sequence (12S, 16S, and COI). Mol. Phylogenet. Evol. 15, 41-58.

Michael, D., Martin, K. C., Seger, R., Ning, M. M., Baston, R., and Kandel, E. R. (1998). Repeated pulses of serotonin required for long-term facilitation activate mitogen-activated protein kinase in sensory neurons of Aplysia. Proc. Natl. Acad. Sci. U.S.A. 95, 1864-1869.

Michel, M., Green, C. L., and Lyons, L. C. (2011a). PKA and PKC are required for long-term but not shortterm in vivo operant memory in Aplysia. Learn. Mem. 18, 19-23.

Michel, M., Green, C. L., Eskin, A., and Lyons, L. C. (2011b). PKGmediated MAPK signaling is necessary for long-term operant memory in Aplysia. Learn. Mem. 18, 108-117. Muller, U., and Carew, T. J. (1998). Serotonin induces temporally and mechanistically distinct phases of persistent PKA activity in Aplysia sensory neurons. Neuron 21, 1423-1434.

Olson, L. M., and Jacklet, J. W. (1985). The circadian pacemaker in the Aplysia eye sends axons throughout the central nervous system. J. Neurosci. 5, 3214-3227.

Plautz, J. D., Kaneko, M., Hall, J. C., and Kay, S. A. (1997). Independent photoreceptive circadian clocks throughout Drosophila. Science 278, 1632-1635.

Raju, U., Koumenis, C., NunezRegueiro, M., and Eskin, A. (1991). Alteration of the phase and period of a circadian oscillator by a reversible transcription inhibitor. Science 253, 673-675.

Raju, U., Yeung, S. J., and Eskin, A. (1990). Involvement of proteins in light resetting ocular circadian oscillators of Aplysia. Am. J. Physiol. 258, R256-R262.

Rawashdeh, O., de Borsetti, N. H., Roman, G., and Cahill, G. M. (2007). Melatonin suppresses nighttime memory formation in zebrafish. Science 318, 1144-1146. 
Reissner, K. J., Shobe, J. L., and Carew, T. J. (2006). Molecular nodes in memory processing: insights from Aplysia. Cell. Mol. Life Sci. 63, 963-974.

Roberts, M., and Block, G. (1982). Dissection of circadian organization of Aplysia through connective lesions and electrophysiological recording. J. Exp. Zool. 219, 39-50.

Rosen, S. C., Teyke, T., Miller, M. W., Weiss, K. R., and Kupfermann, I. (1991). Identification and characterization of cerebral-to-buccal interneurons implicated in the control of motor programs associated with feeding in Aplysia. J. Neurosci. 11, 3630-3655.

Rothman, B. S., and Strumwasser, F. (1976). Phase shifting the circadian rhythm of neuronal activity in the isolated Aplysia eye with puromycin and cycloheximide. Electrophysiological and biochemical studies. J. Gen. Physiol. 68, 359-384.

Rothman, B. S., and Strumwasser, F. (1977). Manipulation of a neuronal circadian oscillator with inhibitors of macromolecular synthesis. Fed. Proc. 36, 2050-2055.

Ruby, N. F., Hwang, C. E., Wessells, C., Fernandez, F., Zhang, P., Sapolsky, R., and Heller, H. C. (2008). Hippocampal-dependent learning requires a functional circadian system. Proc. Natl. Acad. Sci. U.S.A. 105, 15593-15598.

Schwarz, M., and Susswein, A. J. (1986). Identification of the neural pathway for reinforcement of feeding when Aplysia learn that food is inedible. J. Neurosci. 6, 1528-1536.

Sharma, S. K., and Carew, T. J. (2004). The roles of MAPK cascades in synaptic plasticity and memory in Aplysia: facilitatory effects and inhibitory constraints. Learn. Mem. 11, 373-378.

Sharma, S. K., Sherff, C. M., Shobe, J., Bagnall, M. W., Sutton, M. A., and Carew, T. J. (2003). Differential role of mitogen-activated protein kinase in three distinct phases of memory for sensitization in Aplysia. J. Neurosci. 23, 3899-3907.

Strumwasser, F. (1965). "The demonstration and manipulation of a circadian rhythm in a single neuron," in Circadian Clocks, ed. J. Aschoff (Amsterdam: North Holland Publishing Company), 442-462.

Strumwasser, F. (1973). Neural and humoral factors in the temporal organization of behavior. Physiologist 16, 9-42.

Strumwasser, F., Schlechte, F. R., and Bower, S. (1972). Distributed circadian oscillators in the nervous system of Aplysia. Fed. Proc. 31, 405.

Susswein, A. J., Gev, S., Feldman, E., and Markovich, S. (1983). Activity patterns and time budgeting of Aplysia fasciata under field and laboratory conditions. Behav. Neural Biol. 39, 203-220.

Susswein, A. J., and Schwarz, M. (1983). A learned change of response to inedible food in Aplysia. Behav. Neural Biol. 39, 1-6.

Susswein, A. J., Schwarz, M., and Feldman, E. (1986). Learned changes of feeding behavior in Aplysia in response to edible and inedible foods. J. Neurosci. 6, 1513-1527.

Sutton, M. A., and Carew, T. J. (2002). Behavioral, cellular, and molecular analysis of memory in Aplysia I: intermediate-term memory. Integr. Comp. Biol. 42, 725-735.

Sutton, M. A., Masters, S. E., Bagnall, M. W., and Carew, T. J. (2001).
Molecular mechanisms underlying a unique intermediate phase of memory in Aplysia. Neuron 31, 143-154.

Sweatt, J. D. (2010). Mechanisms of Memory, 2nd Edn. San Diego: Elsevier Academic Press.

Takahashi, J. S., Nelson, D. E., and Eskin, A. (1989). Immunocytochemical localization of serotonergic fibers innervating the ocular circadian system of Aplysia. Neuroscience 28, 139-147.

Tononi, G., and Cirelli, C. (2006). Sleep function and synaptic homeostasis. Sleep Med. Rev. 10, 49-62.

Valentinuzzi, V. S., Kolker, D. E., Vitaterna, M. H., Ferrari, E., Takahashi, J. S., and Turek, F. W. (2001). Effect of circadian phase on context and cued fear conditioning in C57BL/6J mice. Anim. Learn. Behav. 29, 133-142.

Villareal, G., Li, Q., Cai, D., and Glanzman, D. L. (2007). The role of rapid, local, postsynaptic protein synthesis in learning-related synaptic facilitation in Aplysia. Curr. Biol. 17, 2073-2080.

Weiss, K. R., Cohen, J. L., and Kupfermann, I. (1978). Modulatory control of buccal musculature by a serotonergic neuron (metacerebral cell) in Aplysia. J. Neurophysiol. 41, 181-203.

Whitmore, D., Foulkes, N. S., and Sassone-Corsi, P. (2000). Light acts directly on organs and cells in culture to set the vertebrate circadian clock. Nature 404, 87-91.

Woodson, P. B., and Schlapfer, W. T (1979). The amplitude of posttetanic potentiation of the EPSP RC1-R15 in Aplysia is modulated by environmental parameters. Brain Res. 173, 225-242.

Wright, W. G., Jones, K., Sharp, P., and Maynard, B. (1995). Widespread anatomical projections of the serotonergic modulatory neuron, $\mathrm{CB} 1$, in Aplysia. Invert. Neurosci. 1, 173-183.

Yeung, S. J., and Eskin, A. (1987). Involvement of a specific protein in the regulation of a circadian rhythm in Aplysia eye. Proc. Natl. Acad. Sci. U.S.A. 84, 279-283.

Ziv, I., Lustig, C., Markovich, S., and Susswein, A. J. (1991a). Sequencing of behaviors in Aplysia fasciata: integration of feeding, reproduction, and locomotion. Behav. Neural Biol. 56, 148-169.

Ziv, I., Lustig, C., Ben-Zion, M., and Susswein, A. J. (1991b). Daily variation of multiple behaviors in Aplysia fasciata: integration of feeding, reproduction, and locomotion. Behav. Neural Biol. 55, 86-107.

Conflict of Interest Statement: The author declares that the research was conducted in the absence of any commercial or financial relationships that could be construed as a potential conflict of interest.

Received: 30 September 2011; paper pending published: 21 November 2011; accepted: 24 November 2011; published online: 05 December 2011.

Citation: Lyons LC (2011) Critical role of the circadian clock in memory formation: lessons from Aplysia. Front. Mol. Neurosci. 4:52. doi: 10.3389/fnmol. 2011.00052

Copyright (c) 2011 Lyons. This is an open-access article distributed under the terms of the Creative Commons Attribution Non Commercial License, which permits non-commercial use, distribution, and reproduction in other forums, provided the original authors and source are credited. 